\title{
Estudio de la investigación turística a través de las coautorías de artículos: cálculo de indicadores de colaboración y análisis de redes sociales. El caso de las universidades catalanas*
}

\author{
José A. Corral-Marfil** \\ Universidad de Vic (España)
}

\section{Ismael M. Rodríguez Herrera*** Ástrid Vargas Vázquez **** \\ Universidad Autónoma de Aguascalientes (México)}

\author{
Gemma Cànoves Valiente $* * * * *$ \\ Universidad Autónoma de Barcelona (España)
}

\begin{abstract}
Resumen: Se analiza la colaboración científica de las universidades catalanas en el campo del turismo entre 2000 y 2010. A partir de las coautorías de artículos, se calculan indicadores de colaboración simples y se examina la red colaboración. Se estudia la colaboración entre investigadores, instituciones y territorios. Los resultados muestran un aumento de la colaboración, diferencias entre las revistas y ausencia de relación entre colaboración y multidisciplinariedad. La red de colaboración presenta una baja densidad y varias subredes.
\end{abstract}

Palabras Clave: turismo; investigación; colaboración científica; coautorías; redes sociales; Cataluña.

\section{Scientific collaboration in tourism: the case of catalan universities}

Abstract: Catalan universities scientific collaboration in tourism is analyzed, from 2000 to 2010 . Through coauthorship data, simple collaboration indicators are calculated and the collaboration network is examined. Collaboration among researchers, institutions and territories is investigated. Results show an increase in collaboration, differences among journals, and absence of relationship between collaboration and multidisciplinarity. The collaboration network presents a low density and several subnetworks.

Keywords: tourism; research; scientific collaboration; coauthorship; social networks; Catalonia.

\section{Introducción y objetivo}

En paralelo al continuo crecimiento del turismo, la investigación turística ha ganado tamaño y reconocimiento en los últimos años. Ha emergido como un dinámico campo de estudio multidisciplinar dentro de las ciencias sociales. Son indicadores de tal dinamismo la proliferación de revistas científicas, la publicación de tesis doctorales, la fundación de centros de investigación y la celebración de congresos

(Faulker, 2003; Racherla y Hu, 2010). En España la academia turística también está creciendo, como muestran el aumento de la producción científica (Albacete y Fuentes, 2010) y la creación de estudios de grado, máster y doctorado (Ceballos et al., 2010).

\footnotetext{
* Este trabajo se ha realizado en el marco de los Estudios de Doctorado en Geografía de la Universidad Autónoma de Barcelona.

** E-mail: jacorralmarfil@gmail.com

*** E-mail: imrodri@correo.uaa.mx

***** E-mail: avargasv@correo.uaa.mx

***** E-mail: gemma.canoves@uab.es
} 
Por otra parte, la colaboración científica ha aumentado durante las últimas décadas en todas las disciplinas (Glänzel y Schubert, 2004). La colaboración, el trabajo en equipo, es una característica de la big science, como lo son las elevadas necesidades de financiación (de Solla Price, 1966). En las ciencias sociales la colaboración es mucho menor que en las ciencias experimentales, aunque algo mayor que en las humanidades (Ardanuy, 2012). La colaboración está presente incluso en las ciencias "menos caras", como las matemáticas puras o la investigación teórica en ciencias sociales.

Beaver (2001) cita 18 razones por que las personas cooperan. Entre ellas, acceder a conocimiento experto, equipo o recursos; obtener prestigio; ganar productividad; progresar más rápidamente; detectar errores más eficientemente; aprender; reducir el aislamiento; pasarlo bien... Sin embargo, la colaboración también comporta costes mayores que el trabajo en solitario (Katz y Martin, 1997). Por ejemplo, por gastos adicionales en desplazamientos, dietas, transporte de equipos; por inversión de tiempo en informar a los miembros del equipo, resolver discrepancias; o por el aumento de la burocracia y los costes de administración.

Por colaboración científica se entiende "la interacción entre dos o más científicos que tiene lugar dentro de un contexto social, la cual permite compartir significado y completar tareas con respecto a una meta superior mutuamente compartida" (Sonnenwald, 2007, p. 645). Se puede clasificar según diversas perspectivas: desde el punto de vista disciplinario (intradisciplinaria, interdisciplinaria, multidisciplinaria), geográfico (presencial, remota, internacional), organizativo y comunitario (universidad-empresa, ciencia-sociedad, triple hélice: universidad-industria-gobierno).

Sonnenwald (2007) sintetiza la extensa literatura sobre colaboración científica. Organiza los factores que influyen en la colaboración según la fase del proceso en que emergen: establecimiento, formulación, mantenimiento y conclusión. Primero, hay factores que pueden impulsar o impedir el establecimiento de colaboraciones. Los hay científicos; políticos; socioeconómicos; relacionados con la accesibilidad a recursos; y con redes sociales y factores personales. Segundo, durante la fase de formulación, los científicos inician y planifican proyectos de investigación. En proyectos colaborativos hay factores que deben considerarse con mayor detalle que en los proyectos de un solo investigador: la visión, los objetivos y las tareas; el liderazgo y la estructura organizativa; las tecnologías de información y la comunicación; la propiedad intelectual y otros temas legales.

Tercero, una vez establecida la colaboración y comenzado el trabajo, hay que mantenerla durante un periodo de tiempo, si se quieren alcanzar los objetivos. Suelen aparecer problemas no previstos que pueden hacer fracasar la colaboración. Para identificar y solucionar los problemas puede ser necesario que evolucionen la estructura organizativa, las tareas, la comunicación y el aprendizaje. En cuarto lugar, idealmente, la colaboración concluye con éxito cuando se alcanzan los objetivos; pero puede haber distintos tipos de resultados considerados exitosos. Por último, se hace difusión de los resultados.

El presente trabajo analiza la colaboración científica de las universidades catalanas en el campo del turismo. Cataluña proporciona un caso relevante para estudiar la investigación turística por el peso que tiene el turismo en la economía y en la ciencia. En efecto, la historia del turismo en Cataluña se remonta al siglo XIX (Garay y Cànoves, 2010). Actualmente es la segunda región de Europa más visitada por turistas extranjeros (Eurostat, 2011). El sector turístico representa alrededor del 11\% del PIB y también del empleo (Direcció General de Turisme, 2011). Y su geografía turística es diversa: tiene turismo litoral, de montaña, urbano y rural (Torres, 2010). Por otra parte, las universidades catalanas son autoras del 20\% de la producción científica sobre turismo de España (González-Albo et al., 2009).

El objetivo del trabajo es estudiar la colaboración a través de las coautorías de artículos. Mediante indicadores de colaboración no relacionales pretende: medir la incidencia y la extensión de la colaboración; descubrir diferencias entre revistas en cuanto al grado de colaboración; explorar la relación entre multidisciplinariedad y colaboración; y analizar la colaboración entre instituciones y entre países. Mediante el análisis de redes sociales, a nivel macro, aspira a describir las características generales de la red de coautorías y medir su centralidad. A nivel micro, determinar la centralidad los principales nodos (autores) de la red. También se propone analizar las redes de colaboración institucional y territorial.

\section{Revisión de la literatura}

\subsection{El estudio de la investigación turística}

Benckendorf (2009) propone dos dimensiones para clasificar las técnicas que se emplean para estudiar un campo de investigación. Primero distingue entre técnicas cualitativas y cuantitativas. La mayoría de las cuantitativas forman parte de la bibliometría. En segundo lugar divide las técnicas 
entre valorativas y relacionales. Las valorativas tienen por objetivo evaluar comparativamente las contribuciones de diversos autores, revistas, instituciones o trabajos académicos. Las relacionales buscan poner de manifiesto relaciones dentro de la investigación científica, como la estructura de los campos de conocimiento. El cruce de ambas dimensiones da lugar a una tipología de cuatro categorías de técnicas, que representan, a su vez, cuatro corrientes de investigación.

Las técnicas cualitativas valorativas consisten en elaborar ránkings de revistas o autores a partir de las opiniones de un grupo o pánel de expertos. Por ejemplo, McKercher et al. (2006) jerarquizan 70 revistas de turismo y hostelería a partir de una encuesta a 505 académicos. Por otra parte, mediante técnicas cuantitativas valorativas se analiza la producción científica en un campo de conocimiento o en una disciplina científica para evaluar la contribución de instituciones, revistas o autores. A menudo se basan en recuentos de artículos o citas, pueden considerar factores de impacto y despiertan mucha controversia. Por ejemplo, Zhao y Ritchie (2007) identifican los 57 investigadores más prolíficos en el campo del turismo en función de la cantidad de artículos publicados en ocho revistas entre 1985 y 2004.

En tercer lugar, a través de técnicas cualitativas relacionales se exploran temas clave en la literatura y tendencias, así como el uso de metodologías y técnicas estadísticas. Aquí Benckendorf (2009) incluye las revisiones y puesta al día, los mapas conceptuales, los análisis de contenido y los metaanálisis. Por ejemplo, Xiao y Smith (2006) identifican las 27 áreas temáticas principales en la investigación turística a lo largo de 30 años, a partir del análisis del índice de materias de la revista Annals of Tourism Research.

Y en cuarto lugar, a través de técnicas cuantitativas relacionales se calculan indicadores bibliométricos para estudiar la investigación más allá de la medida de la productividad y la elaboración ránkings. Incluyen los análisis de coocurrencias de términos (en el título o las palabras clave), de citas (a autores o artículos), cocitas, coautorías y redes sociales. Por ejemplo, Barrios et al. (2008) estudian la evolución de la producción sobre psicología del turismo entre 1990 y 2005; comprueban las leyes bibliométricas de Price, Lotka y Bradford; y analizan la distribución de las citas y su relación con la colaboración. Constatan que los artículos con más de un autor reciben más citas que los firmados por un solo autor.

Pero la mayoría de estudios sólo contemplan revistas turísticas, cuando una parte considerable de la literatura se publica en revistas no turísticas. Hasta hace poco ha habido pocas revistas turísticas de reconocido prestigio internacional (Chang y McAleer, 2012). Como afirma Sancho: "la escasez de revistas dedicadas al turismo, con validez y reconocimiento para la evaluación de la actividad investigadora, hace que las investigaciones en turismo queden dispersas en un abanico de revistas de diferentes disciplinas" (Pulido, 2006, p. 57). El presente estudio se enmarca en la cuarta corriente; aquélla que investiga el contenido, la estructura y la evolución de la investigación turística con el fin de contribuir a su desarrollo. El trabajo analiza la colaboración científica en turismo cuantitativamente, sin pretender elaborar ránkings, y contempla revistas turísticas y no turísticas.

\subsection{El estudio de la colaboración en turismo}

Existen pocos trabajos que hayan investigado la colaboración científica en turismo (Racherla y Hu, 2010). En el ámbito internacional, destacan tres estudios: Ye, Li y Law (2012), Benckendorff (2010) y Racherla y Hu (2010). Los tres analizan la colaboración a partir de las coautorías de artículos y los tres emplean el análisis de redes sociales.

Ye, Li y Law (2012) investigan la colaboración entre académicos. Construyen la red social de coautorías a partir de los artículos publicados en seis importantes revistas de turismo y hostelería entre 1991 y 2010. A nivel macro, analizan las características y la evolución de la red en su conjunto y, a nivel micro, evalúan investigadores individualmente. A nivel macro, muestran que la red de colaboración es una red de "mundo pequeño". Esto es, una red en la que la mayoría de nodos tienen pocos vecinos y, sin embargo, en pocos pasos pueden alcanzar cualquier otro nodo de la red (Molina, 2004). También comparan los atributos de la red con los de otras redes de otras disciplinas. A nivel micro, identifican nodos centrales de la red, que son nodos con muchas conexiones o nodos que enlazan subgrupos. Además exploran la relación entre la colaboración y la productividad científica.

Referido a Australia y Nueva Zelanda, Benckendorff (2010) estudia la colaboración científica en el campo del turismo entre investigadores, instituciones y países. El estudio se basa en las coautorías de artículos publicados por investigadores australianos y neozelandeses en 18 revistas turísticas, entre 1999 y 2008 . Mediante el cálculo de indicadores bibliométricos, analiza la incidencia, extensión y evolución de la colaboración. Y mediante el análisis de redes sociales, explora las pautas generales de colaboración. Los resultados indican un aumento de la incidencia y la extensión de la colaboración entre autores, y también un incremento de la colaboración internacional. La red social de coautorías 
está dispersa y poco cohesionada. Tiene muchos autores y grupos desconectados y está dominada por un grupo o componente principal.

Racherla y Hu (2010) estudian los patrones de colaboración entre los académicos del turismo mediante el análisis de redes sociales, identifican investigadores clave y presentan la evolución de varios atributos de la red de coautorías. La base empírica del estudio son los artículos publicados entre 1996 y 2005 en las tres revistas top: Annals of Tourism Research, Journal of Travel Research y Tourism Management. Constatan un incremento en la colaboración y la existencia de unos pocos autores con muchas colaboraciones y muchos autores con muy pocas. Observan que la red de coautorías está muy poco cohesionada. Por otra parte, detectan los autores con mayor centralidad (medida por su grado, cercanía e intermediación) y exploran la relación entre centralidad y productividad.

En España, hasta donde conocen los autores, casi no se ha estudiado la colaboración científica en el campo del turismo. Y sólo González-Albo et al. (2009) emplean, limitadamente, el análisis de redes sociales. Sánchez y Marín (2003) analizan los artículos publicados en las revistas Papers de Turisme y Estudios Turísticos entre 1996 y 2001. Calculan el índice de coautoría y el porcentaje de colaboración. Además detallan ambos indicadores según áreas de conocimiento de la economía de la empresa, nacionalidad de los autores, nacionalidad de las universidades y enfoque empírico o conceptual. Por otra parte, Hernández et al. (2011) tocan tangencialmente el tema de la colaboración al analizar los estudios publicados sobre gestión turística en las revistas españolas de dirección de empresas. Comentan la distribución del número de autores por artículo.

González-Albo et al. (2009) analizan múltiples dimensiones de la producción científica sobre turismo en España. Mediante una estrategia de búsqueda basada en palabras clave, recogen los documentos publicados en revistas turísticas y no turísticas entre 1998 y 2008. Dividen el estudio en dos partes, según la fuente de la que recogen los datos: la base de datos internacional Web of Science y la española ISOC de ciencias sociales y humanidades. Calculan indicadores de colaboración como el índice de coautoría y la colaboración inter-centros, nacional e internacional, así como su evolución. También describen la intensidad de colaboración entre comunidades autónomas de España y entre España y otros países.

El presente estudio extiende estos análisis. Se centra en el análisis de la colaboración y emplea tanto indicadores de colaboración simples como el análisis de redes sociales. En el siguiente apartado explica la metodología seguida. A continuación se presentan los resultados obtenidos. Y finalmente se extraen unas conclusiones.

\section{Metodología}

La colaboración científica en turismo de las universidades catalanas se analizó mediante un estudio bibliométrico. La base empírica fueron los artículos sobre turismo publicados en revistas científicas, entre 2000 y 2010 , firmados al menos por un autor afiliado a alguna de las doce universidades catalanas. La estrategia de búsqueda de artículos se basó en dos listas de términos relacionados con el turismo, una en español y la otra en inglés. Las listas se elaboraron añadiendo algunos términos a los glosarios empleados por González-Albo et al. (2009), como excursio*, aloja * o peregrin *. La ventaja de la búsqueda mediante listas de términos es que permite alcanzar revistas no turísticas y, por tanto, es más exhaustiva que la búsqueda basada en una selección de revistas turísticas.

Las fuentes de información fueron cuatro bases de datos bibliográficas. Los artículos publicados en revistas españolas se recolectaron de ISOC (ciencias sociales y humanidades) y de ICYT (ciencia y tecnología), ambas del Centro de Ciencias Humanas y Sociales del CSIC. Los artículos de revistas extranjeras se buscaron en Scopus de Elsevier y en CAB Direct de CABI. En CAB Direct se buscó dentro de los códigos temáticos (cabicodes) Tourism and Travel (UU700) y Leisure, Recreation and Tourism Economics (EE119). La combinación de las cuatro bases de datos permitió alcanzar la mayoría de revistas científicas reconocidas, nacionales e internacionales, tanto turísticas como no específicamente turísticas.

Las búsquedas dieron como resultado los 429 artículos, de los cuales se codificaron los atributos: autores, afiliación de los autores (institución, comunidad autónoma y país), revista, tipo de revista (indexada en los Journal Citacion Reports —JCR— de Thomson ISI, indexada en Scopus), disciplina científica de la revista y disciplina científica del artículo. Los datos se analizaron, mediante el cálculo de indicadores bibliométricos de colaboración no relacionales y mediante un análisis de redes sociales.

Para analizar la red de coautorías, a partir de la base de datos, se construyeron una serie de matrices que medían la colaboración y consideraban algunos atributos: producción, origen de los autores, universidad de adscripción, etc. Se grabó la información en una hoja de cálculo de Microsoft 
Excel. Después se exportó al programa Ucinet 6 y se generaron grafos con el software complementario NetDraw 9.091. Al construir los grafos, se ocultaron los nodos sin colaboración, para "limpiar" el grafo y facilitar su análisis.

El análisis de redes sociales adoptó dos perspectivas: las relaciones entre los autores y entre sus instituciones. En ambos casos, los niveles de análisis fueron global o macro, de la red en general; e individual o micro (Benckendorff, 2010; Ye et al., 2011). El software Ucinet 6 permitió obtener una serie de índices estadísticos vinculados a las medidas de centralidad propuestas por Freeman (1979). A nivel macro se consideraron la densidad y los grados de centralización e intermediación de la red, mientras que a nivel micro se analizaron el grado de centralidad y la intermediación para cada uno de los nodos. Adicionalmente, llevó a cabo un análisis a partir de la localización geográfica de las instituciones.

\section{Resultados}

\subsection{Indicadores de colaboración no relacionales}

\subsubsection{Porcentaje de colaboración e índice de coautoría}

A lo largo del decenio 2000-2010 se publicaron 429 artículos. El 40,1\% de los artículos fue firmado por un solo autor y el 59,9\%, por varios autores. Del primer al segundo quinquenio, el porcentaje de artículos escritos en colaboración aumentó del 51\% al 65\%. El Gráfico 1 muestra la evolución del número anual de artículos escritos en colaboración y en solitario.

\section{Gráfico 1: Evolución del número anual de artículos escritos en solitario y en colaboración}

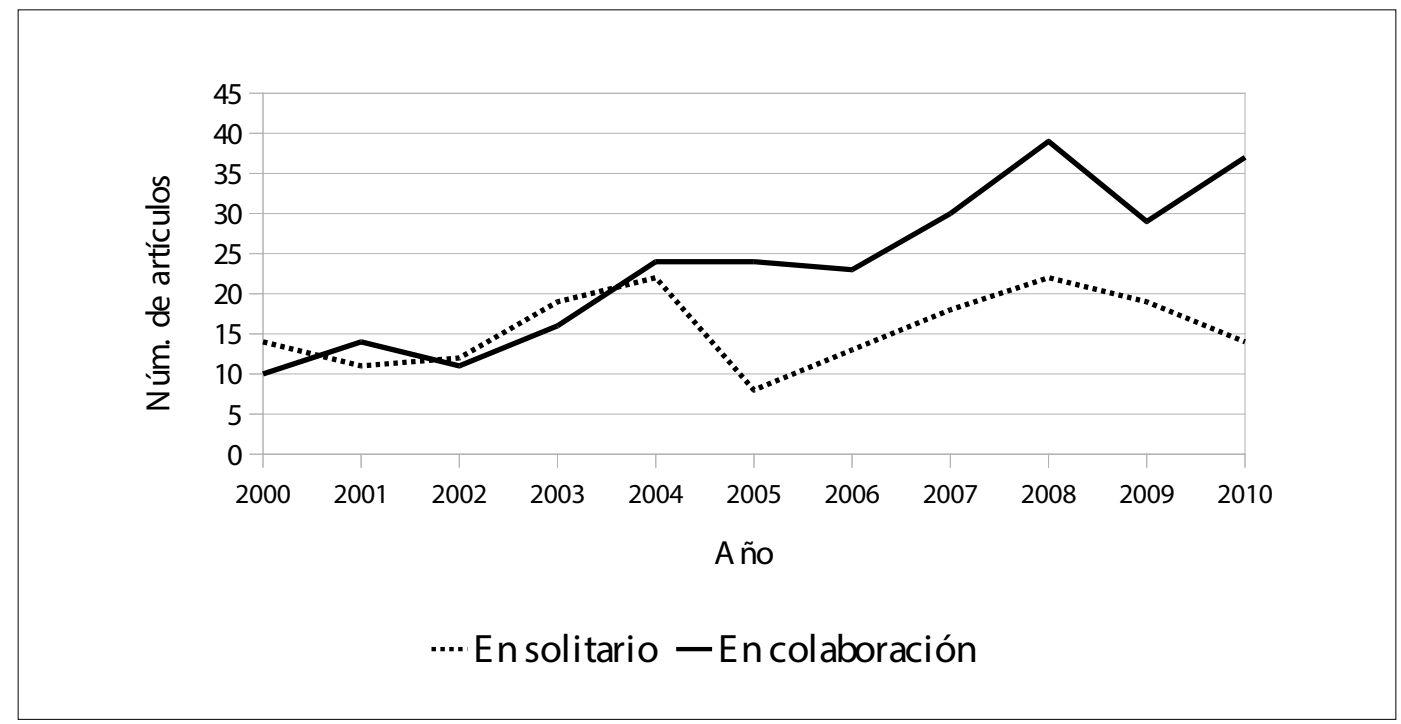

Fuente: elaboración propia

Dos fue el número de autores más frecuente en los trabajos escritos en colaboración. Casi la mitad de los trabajos fueron firmados por dos o tres autores (49\%). Y los trabajos firmados por más de cuatro autores fueron raros (4\%) (Tabla 1). Puesto que en los 429 artículos hubo 884 firmas, la media aritmética o índice de coautoría fue 2,06 firmas por artículo; la moda fue una firma; la mediana, dos; el máximo, 11; y la desviación típica, 1,37 firmas. El índice de coautoría no varió significativamente a lo largo de la década: de dos autores por artículo en el primer lustro se pasó a 2,10 en el segundo. 
Tabla 1: Distribución de los artículos según el número de firmas

\begin{tabular}{|c|c|c|c|c|c|c|}
\hline $\begin{array}{c}\text { Núm. de } \\
\text { firmas }\end{array}$ & \multicolumn{2}{|c|}{ Núm. de artículos } & \multicolumn{2}{|c|}{ \% de artículos } & \multicolumn{2}{c|}{ \% acumulado } \\
\hline 1 & 172 & & 40,1 & & 40,1 & \\
\hline 2 & 152 & & 35,4 & & 75,5 & \\
\hline 3 & 58 & & 13,5 & & 89,0 & \\
\hline 4 & 30 & & 7,0 & & 96,0 & \\
\hline 5 & 8 & & 1,9 & & 97,9 & \\
\hline 6 & 3 & & 0,7 & & 98,6 & \\
\hline 8 & 2 & & 0,5 & & 99,1 & \\
\hline 9 & 2 & & 0,5 & & 99,5 & \\
\hline 11 & 2 & & 0,5 & & 100,0 & \\
\hline Total & 429 & & 100,0 & & & \\
\hline
\end{tabular}

Fuente: elaboración propia

\subsubsection{La colaboración según revistas}

La Tabla 2 muestra el porcentaje de colaboración y el índice de coautoría de las revistas con al menos 5 artículos. Se observan diferencias notorias entre las revistas en estos indicadores.

\section{Tabla 2: Índice de coautoría y porcentaje de colaboración} de las revistas con más artículos

\begin{tabular}{|c|c|c|c|c|c|c|c|c|}
\hline Revista & $\begin{array}{l}\text { Núm. de } \\
\text { artículos }\end{array}$ & $\begin{array}{l}\% \text { de } \\
\text { artíc. }\end{array}$ & $\%$ acum. & $\begin{array}{l}\% \text { de } \\
\text { colab. }\end{array}$ & Í.C. & $\begin{array}{l}\text { Desv. } \\
\text { típ I.C. }\end{array}$ & Moda & Máximo \\
\hline Estudios Turísticos & 22 & 5,1 & 5,1 & 50,0 & 1,77 & 0,92 & 1 & 4 \\
\hline $\begin{array}{c}\text { Estudis de Turisme de } \\
\text { Catalunya }\end{array}$ & 18 & 4,2 & 9,3 & 22,2 & 1,28 & 0,57 & 1 & 3 \\
\hline Cuadernos de Turismo & 16 & 3,7 & 13,1 & 75,0 & 2,06 & 0,85 & 2 & 4 \\
\hline PASOS & 16 & 3,7 & 16,8 & 56,3 & 1,63 & 0,62 & 2 & 3 \\
\hline Tourism Management & 13 & 3,0 & 19,8 & 76,9 & 2,23 & 1,09 & 2 & 5 \\
\hline $\begin{array}{c}\text { Documents d'Anàlisi } \\
\text { Geogràfica }\end{array}$ & 11 & 2,6 & 22,4 & 63,6 & 1,64 & 0,50 & 2 & 2 \\
\hline Papers de Turisme & 11 & 2,6 & 24,9 & 63,6 & 1,73 & 0,65 & 2 & 3 \\
\hline Scripta Nova & 11 & 2,6 & 27,5 & 18,2 & 1,18 & 0,40 & 1 & 2 \\
\hline Boletín de la AGE & 9 & 2,1 & 29,6 & 66,7 & 2 & 0,87 & $1,2,3$ & 3 \\
\hline Iber & 9 & 2,1 & 31,7 & 22,2 & 1,22 & 0,44 & 1 & 2 \\
\hline Tourism Economics & 9 & 2,1 & 33,8 & 100,0 & 2,89 & 1,05 & 2 & 5 \\
\hline Revista de Análisis Turístico & 7 & 1,6 & 35,4 & 71,4 & 2,29 & 1,70 & 2 & 6 \\
\hline $\begin{array}{c}\text { Cuadernos Geográficos Univ. } \\
\text { Granada }\end{array}$ & 6 & 1,4 & 36,8 & 66,7 & 2,17 & 1,17 & 1,2 & 4 \\
\hline $\begin{array}{c}\text { Annals of Tourism Research en } \\
\text { Español }\end{array}$ & 5 & 1,2 & 38,0 & 80,0 & 3,40 & 1,82 & 5 & 5 \\
\hline Investigaciones Geográficas & 5 & 1,2 & 39,2 & 60,0 & 2,00 & 1,00 & 1,3 & 3 \\
\hline $\begin{array}{c}\text { Revista Aragonesa de Admón. } \\
\text { Pública }\end{array}$ & 5 & 1,2 & 40,3 & 100,0 & 2,40 & 0,55 & 2 & 3 \\
\hline Tourism Review & 5 & 1,2 & 41,5 & 100,0 & 2,20 & 0,45 & 2 & 3 \\
\hline 181 revistas restantes & 251 & 58,5 & 100,0 & 60,6 & 2,19 & 1,58 & 1 & 11 \\
\hline Total & 429 & 100,0 & & 59,9 & 2,06 & 1,37 & 1 & 11 \\
\hline
\end{tabular}

Fuente: elaboración propia 
El Gráfico 2 muestra los porcentajes de colaboración respectivos de los artículos publicados en revistas indexadas y no indexadas en las bases de datos del ISI Web of Science ${ }^{1}$. En revistas no indexadas por el ISI, el 54,2\% de los artículos se escribió en colaboración, mientras que en revistas sí indexadas, el $80 \%$. Es decir, el porcentaje de colaboración fue significativamente mayor en las revistas indexadas. De igual forma, el porcentaje de colaboración fue significativamente más grande en los artículos de las revistas indexadas en Scopus ${ }^{2}$ (Gráfico 3).

También el índice de coautoría fue significativamente mayor en los artículos publicados en revistas indexadas que en las no indexadas. El índice de coautoría en los artículos publicados en revistas ISI fue 2,73 firmas por trabajo, frente a las 1,87 firmas por trabajo en revistas no ISI. El índice de coautoría de los artículos publicados en revistas Scopus fue 2,52 firmas por trabajo, frente a 1,82 firmas en revistas no Scopus.

\section{Gráficos 2 y 3: Porcentajes de colaboración según indexación de las revistas}
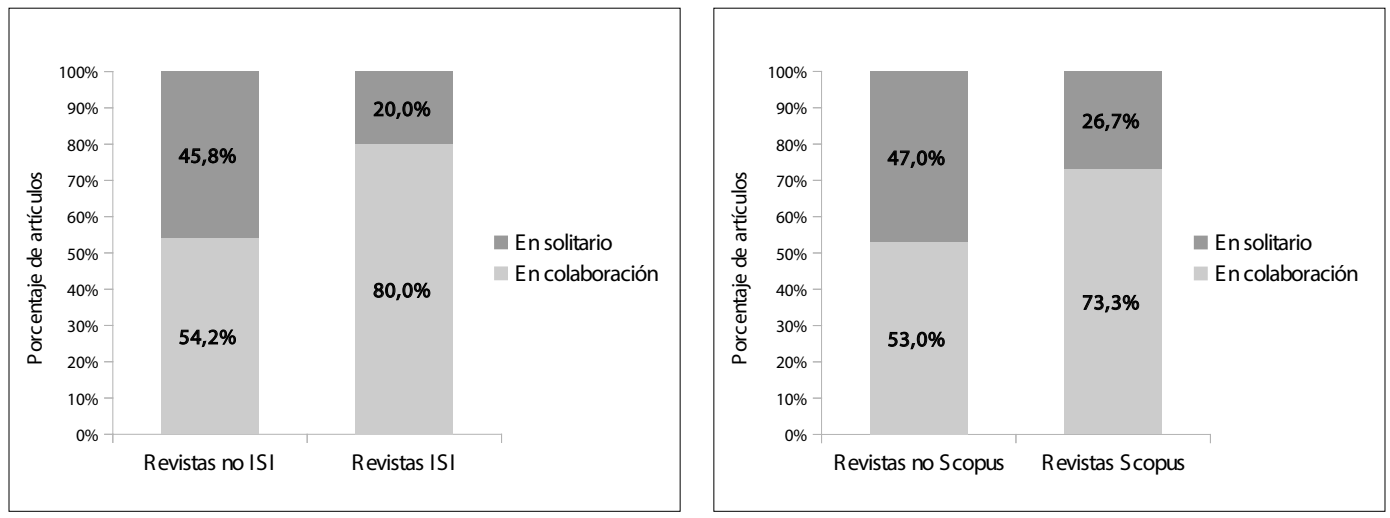

Fuente: elaboración propia

\subsubsection{Multidisciplinariedad y colaboración}

Con el fin de explorar la incidencia de la multidisplinariedad sobre la colaboración, se cruzaron variables indicadoras del grado de colaboración (índice de coautoría y porcentaje de colaboración) con variables relacionadas con el número de disciplinas de los artículos y las revistas.

La segunda y tercera columnas de la Tabla 3 contienen el índice de coautoría y el porcentaje de colaboración de los artículos publicados en revistas españolas, respectivamente. La diferencias que se produjeron en estos indicadores de colaboración entre los artículos unidisciplinares y los multidisciplinares fueron mínimas y estadísticamente no significativas al 95\% de confianza. La cuarta y quinta columnas de la Tabla 3 muestran los mismos indicadores, pero referidos a las disciplinas científicas de las revistas. En este caso sí hubo diferencias. Sin embargo, las diferencias fueron en el sentido opuesto al previsto. Es decir, hubo más colaboración en las revistas unidisciplinares que en las multidisciplinares.

Tabla 3: Indicadores de la relación entre multidisciplinariedad y colaboración

\begin{tabular}{|c|c|c|c|c|c|c|}
\hline & \multicolumn{3}{|c|}{ REVISTAS ESPANOLAS ISOC } & \multicolumn{2}{c|}{ REVISTAS SCOPUS } \\
\hline & \multicolumn{2}{|c|}{ Artículos $^{3}$} & \multicolumn{2}{c|}{ Revistas $^{4}$} & \multicolumn{2}{c|}{ Revistas $^{5}$} \\
\hline Núm. de disciplinas & I.C. & $\%$ Colab. & Í.C. & $\%$ Colab. & I.C. & $\%$ Colab. \\
\hline Una disciplina & 1,82 & 54,1 & 1,89 & 55,4 & 2,22 & 65,9 \\
\hline Más de una disciplina & 1,92 & 53,8 & 1,55 & 32,5 & 2,64 & 76,2 \\
\hline Total & 1,87 & 53,9 & 1,84 & 52,4 & 2,52 & 73,3 \\
\hline
\end{tabular}

Fuente: elaboración propia 
Y referente a las revistas indexadas en Scopus, españolas y extranjeras, las disciplinas de las revistas se obtuvieron de los All Science Classification Codes (ASJC). La sexta y séptima columnas de la Tabla 3 presentan respectivamente el índice de coautoría y el porcentaje de colaboración de las revistas indexadas en Scopus. Aunque hubo diferencias en los indicadores de colaboración entre las revistas unidisciplinares y las multidisciplinares, éstas no fueron estadísticamente significativas.

\subsubsection{Colaboración institucional y territorial}

La Tabla 4 muestra cómo se distribuyeron los artículos según el número de instituciones participantes. El 69\% de los trabajos fueron firmados por autores afiliados a una misma institución, es decir, sólo hubo colaboración institucional en el 31\% de los artículos. En promedio participaron 1,42 instituciones por artículo (desviación típica: 0,87).

\section{Tabla 4: Distribución del número de instituciones colaboradoras por trabajo}

\begin{tabular}{|c|c|c|c|c|c|c|}
\hline $\begin{array}{c}\text { Número de } \\
\text { instituciones }\end{array}$ & \multicolumn{2}{|c|}{$\begin{array}{c}\text { Núm. de } \\
\text { artículos }\end{array}$} & \multicolumn{2}{c|}{ \% De artículos } & \multicolumn{2}{c|}{$\begin{array}{c}\text { \% } \\
\text { Acumulado }\end{array}$} \\
\hline 1 & 296 & & 69,0 & & 69,0 & \\
\hline 2 & 108 & & 25,2 & & 94,2 & \\
\hline 3 & 16 & & 3,7 & & 97,9 & \\
\hline 4 & 4 & & 0,9 & & 98,8 & \\
\hline 5 & 2 & & 0,5 & & 99,3 & \\
\hline 6 & 1 & & 0,2 & & 99,5 & \\
\hline 7 & 1 & & 0,2 & & 99,8 & \\
\hline 11 & 1 & & 0,2 & & 100,0 & \\
\hline Total & 429 & & 100,0 & & & \\
\hline
\end{tabular}

Fuente: elaboración propia

La colaboración científica puede clasificarse en tres tipos o niveles: local, doméstica e internacional (Ardanuy, 2012). La colaboración local se produce cuando todos los investigadores trabajan en la misma institución. En la doméstica participan investigadores de diferentes instituciones de un mismo país. Y en la internacional intervienen colaboradores de distintos países. La Tabla 5 muestra la distribución según niveles de las colaboraciones sobre turismo de los investigadores de las universidades catalanas. ${ }^{6}$ Casi la mitad de las colaboraciones (48,2\%) fueron locales, es decir, entre autores de la misma institución.

Tabla 5: Distribución de las colaboraciones según niveles

\begin{tabular}{|c|c|c|}
\hline Nivel de colaboración & $\begin{array}{l}\text { Núm } \\
\text { artíc }\end{array}$ & $\begin{array}{r}\% D \\
\text { artícu }\end{array}$ \\
\hline Sin colaboración: un único autor & 172 & 40,1 \\
\hline Colaboración local: varios autores de la misma institución & 124 & 28,9 \\
\hline Colaboración catalana: autores de diferentes instituciones de Cataluña & 47 & 11,0 \\
\hline Colaboración española: autores de instituciones de Cataluña y de España & 35 & 8,2 \\
\hline Colaboración internacional: autores de instituciones de diferentes países & 51 & 11,9 \\
\hline Total & 429 & 100,0 \\
\hline
\end{tabular}

Fuente: elaboración propia 
El Gráfico 4 presenta la evolución a lo largo del decenio de los tres tipos de colaboraciones. Comparando el primer lustro con respecto al segundo, la colaboración que más creció fue la doméstica, que casi se triplicó, mientras que la internacional se duplicó, aproximadamente. La colaboración local, a pesar de ser la más frecuente (Tabla 5), fue la que menos aumentó, multiplicándose por 1,5.

\section{Gráfico 4: Evolución de los tres tipos de colaboración}

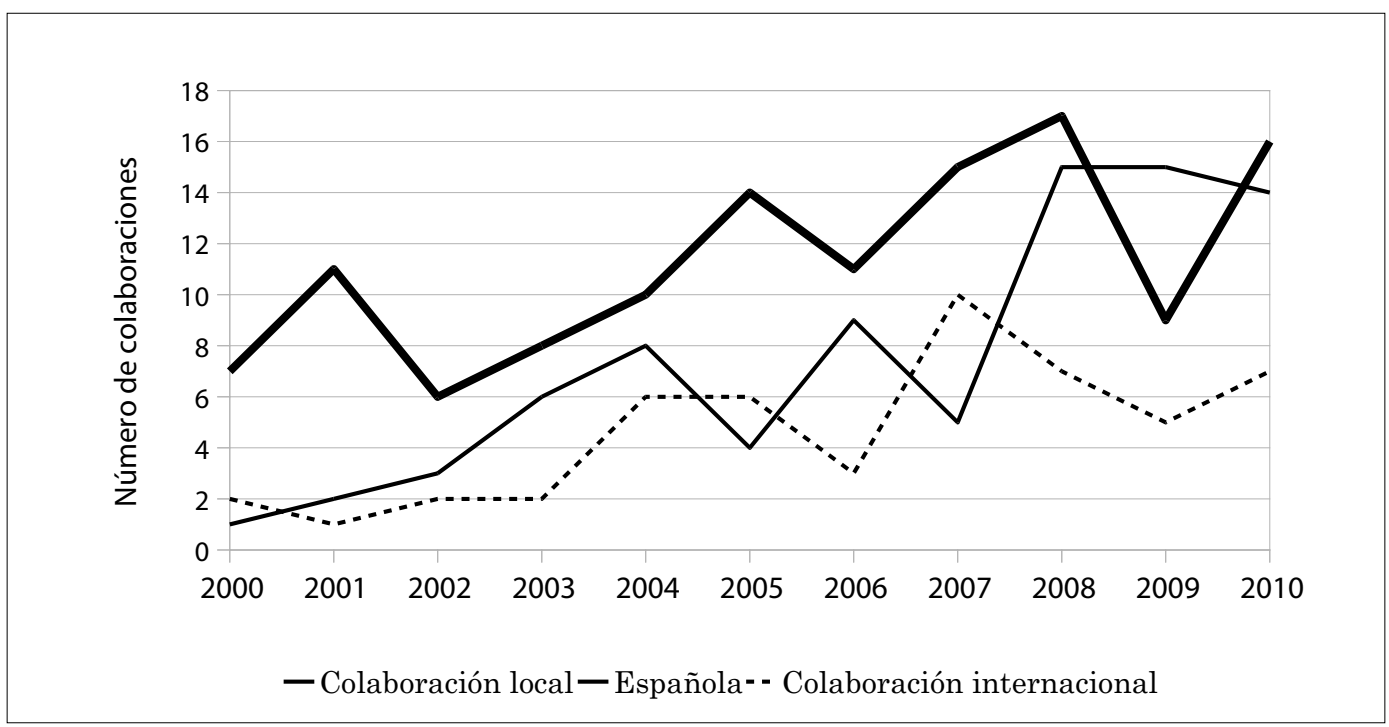

Fuente: elaboración propia

Dentro de la colaboración española (Tabla 5), las cuatro comunidades autónomas con las que Cataluña colaboró más frecuente fueron las Islas Baleares $\left(24 \%{ }^{7}\right)$, Galicia (18\%), Andalucía (16\%) y la Comunidad Valenciana (13,3\%). Además, aunque menos frecuentemente, Cataluña colaboró con otras ocho comunidades autónomas (porcentajes inferiores al 10\%). Por otra parte, dentro de la colaboración internacional, los cuatro países con los que más colaboró Cataluña fueron el Reino Unido $\left(14 \%^{8}\right)$, Holanda (13\%), Italia (11\%) y Estados Unidos (10\%). Adicionalmente, Cataluña colaboró, pero menos, con otros 23 países.

\subsection{Análisis de redes sociales}

El estudio de la colaboración mediante el análisis de redes sociales se efectuó desde tres perspectivas: autores, instituciones y ubicación de éstas; y a dos niveles: macro y micro.

\subsubsection{La red de coautores}

El Gráfico 5 muestra la red para los 257 artículos escritos por los 346 autores que publicaron al menos un artículo en coautoría. Los nodos representan autores, las líneas que conectan los nodos muestran coautorías entre ellos y el grosor de las líneas indica el número de publicaciones conjuntas. Además, el tamaño del nodo es proporcional al número de artículos publicados (atributo producción).

A nivel macro se observa una red no conexa con varias subredes. Las medidas de centralidad dan cuenta una baja interrelación entre nodos, pues la densidad fue de 0,0067, que es resultado de dividir el número de relaciones (coautorías) existentes entre el total de posibles relaciones. Además, se obtuvo un grado de centralidad media para los actores de 3,417, lo cual implica que cada autor tuvo en promedio tres o cuatro coautorías con otros autores. El grado de centralización fue del 0,68\% y la intermediación media, 13,844. Esto último hace referencia al número de veces que un autor fue intermediario entre otros dos autores. 


\section{Gráfico 5: Red de colaboración entre autores}

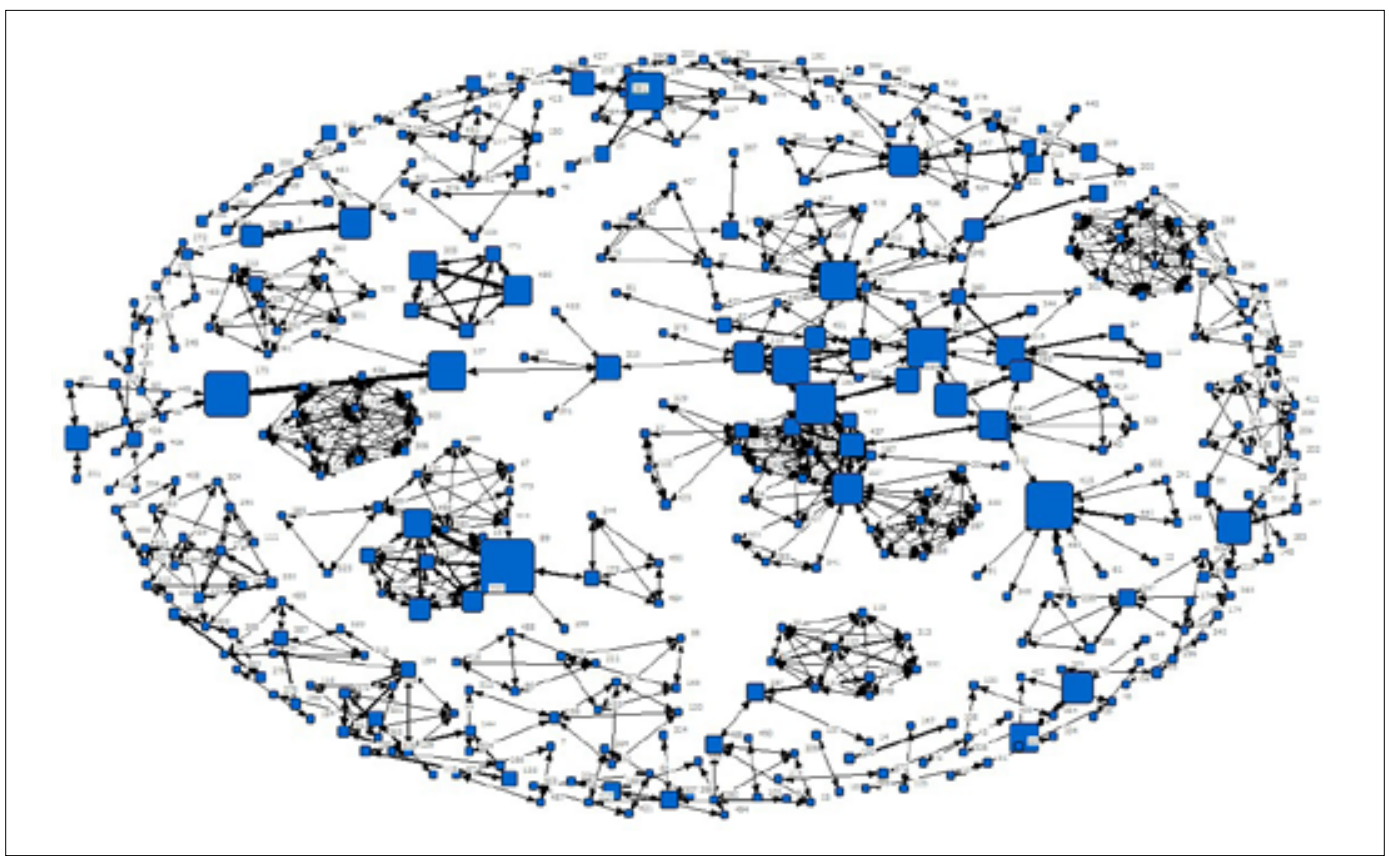

Fuente: elaboración propia

Para llevar a cabo el análisis a nivel micro, se elaboró la Tabla 6. Contiene la lista de autores con mayor número de relaciones (mayor vecindario), así como dos medidas de centralidad: el grado de centralidad (número de colaboraciones por autor) y la intermediación (número de veces que un autor fue intermediario entre otros dos investigadores). Las medidas de centralidad pueden ser entendidas como la importancia del autor, su influencia y la capacidad que tiene de acceder a otros autores dentro de la red (Benckendorff, 2010).

Tabla 6: Medidas de colaboración de 11 autores

$\begin{array}{cccccc}\text { Id } & \text { Autor } & \text { Artículos } & \text { Centralidad } & \text { Intermediación } & \text { Colaboradores } \\ 227 & \text { Jiménez, José A. } & 8 & 31 & 0,460 & 20 \\ 18 & \text { Anton, Salvador } & 9 & 16 & 0,390 & 16 \\ 437 & \text { Sardà, Rafael } & 6 & 20 & 0,196 & 13 \\ 394 & \text { Roca, Elisabet } & 3 & 15 & 0,155 & 13 \\ 161 & \text { Fluvià, Modest } & 11 & 26 & 0,709 & 13 \\ 23 & \text { Ariza, Eduardo } & 4 & 18 & 0,021 & 12 \\ 167 & \text { Fraguell, Rosa M. } & 2 & 11 & 0,046 & 11 \\ 497 & \text { Villares, Míriam } & 2 & 12 & 0,021 & 10 \\ 477 & \text { Valdemoro, } & 3 & 12 & 0,000 & 10 \\ 415 & \text { Husso, Antonio P. } & 13 & 12 & 0,260 & 10 \\ 89 & \text { Cànoves, Gemma } & 15 & 28 & 0,037 & 9\end{array}$

Fuente: elaboración propia 
En este caso destacan los primeros cinco autores enlistados. Debido a que tuvieron un número importante de colaboradores y una producción de artículos significativa, tuvieron medidas de centralidad elevadas, tanto en el grado de centralidad como en la intermediación. Fueron autores brokers, investigadores clave, que mantuvieron la cohesión de la red y funcionaron como enlaces o puentes entre los demás nodos. Se deben destacar los autores 89 y 415 . El primero mostró un grado elevado de centralidad y el segundo, de intermediación. Por tanto, fueron actores con roles importantes en la colaboración y la intermediación de la red.

Hay que aclarar que se excluyeron de la Tabla 6 un número considerable de autores que, aunque tuvieron un número importante de colaboradores (10), sólo publicaron un trabajo en coautoría. Además, otros autores no aparecen en la Tabla 6 por su reducido vecindario, aunque tuvieron un alto grado de centralidad $(498,143,480,110,355,220,53,118$ o 213) o de intermediación (110, 310, 417, 317, 403, 137, 394. 213, 274, 170, 481 о 41).

\subsubsection{Red institucional}

El análisis institucional fue similar al de los autores, salvo que los nodos ahora representan instituciones. Pero las líneas representan igualmente colaboración entre nodos y su grosor denota intensidad en la colaboración. La red incluye los artículos publicados por 116 instituciones. Está algo más conectada que la de autores (Gráfico 6). En este caso, la densidad fue de 0,043, lo que indica una cohesión también baja y que la red de instituciones está dispersa. El grado de centralidad media fue de 5,052 , lo que significa que, en promedio, cada institución mantuvo poco más de cinco colaboraciones. El grado de centralización de la red fue del 5,19\% y la intermediación media, 211.379.

\section{Gráfico 6: Red de colaboración entre instituciones}

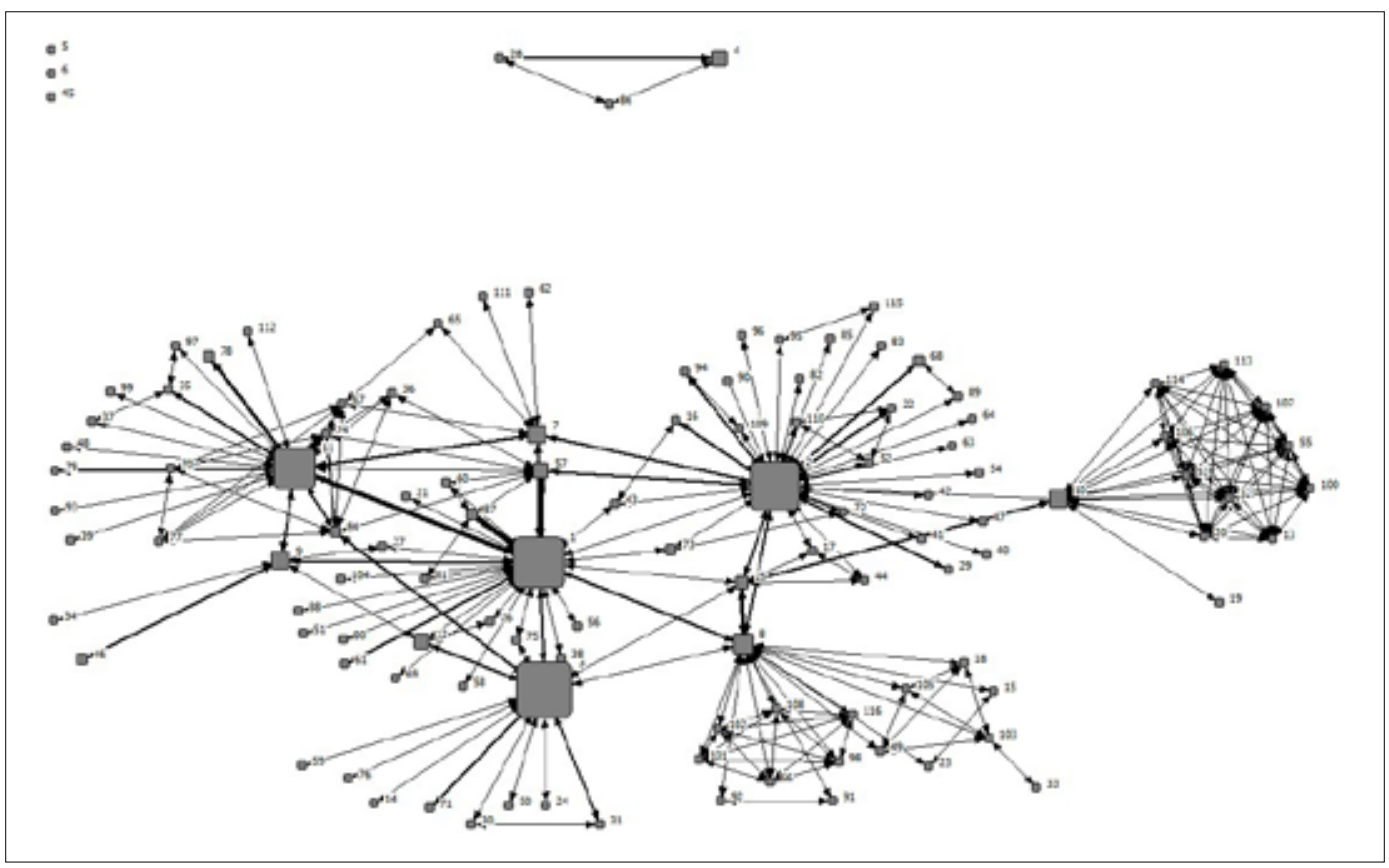

Fuente: elaboración propia

El grafo da cuenta de una red en la que existieron diferentes subgrupos. Pero destacaron seis instituciones que, por su mayor número de colaboradores y artículos publicados, tuvieron un rol fundamental en la conectividad de la red. Lo anterior se corrobora con los datos contenidos en la Tabla 7. 
Tabla 7: Medidas de colaboración de 11 instituciones

\begin{tabular}{cccccc}
\hline Id. & Institución & Artículos & Centralidad & Intermediación & Colaboradores \\
2 & UB & 129 & 45 & 40,366 & 32 \\
1 & UAB & 136 & 52 & 38,243 & 27 \\
11 & URV & 105 & 36 & 18.419 & 20 \\
8 & UPC & 41 & 24 & 23,648 & 18 \\
3 & UdG & 151 & 21 & 14,335 & 14 \\
10 & URL & 29 & 14 & 16,514 & 13 \\
57 & UIB & 20 & 16 & 5,043 & 8 \\
84 & U. Jaume I & 5 & 11 & 2,487 & 8 \\
7 & UOC & 31 & 10 & 7,407 & 7 \\
25 & CEAB-CSIC & 12 & 12 & 13,883 & 7 \\
9 & UPF & 27 & 9 & 3,496 & 6 \\
\hline
\end{tabular}

Fuente: elaboración propia

\subsubsection{Red territorial}

Por último, se realizó un análisis de redes a partir del atributo sobre la ubicación de las universidades de adscripción de los autores (Gráfico 7). Destacó la relación con los nodos 27 (Italia), 18 (Estados Unidos), 36 (Reino Unido), 25 (Islas Baleares), 2 (Andalucía), 23 (Holanda) y 13 (Comunidad Valenciana). A nivel macro, se observa una red mucho más conectada, con una densidad de 0,247 , un grado de centralidad media de 9,650 y un grado de centralización de la red de $25,85 \%$.

\section{Gráfico 7: Red de colaboración entre Cataluña y otras comunidades autónomas y países}

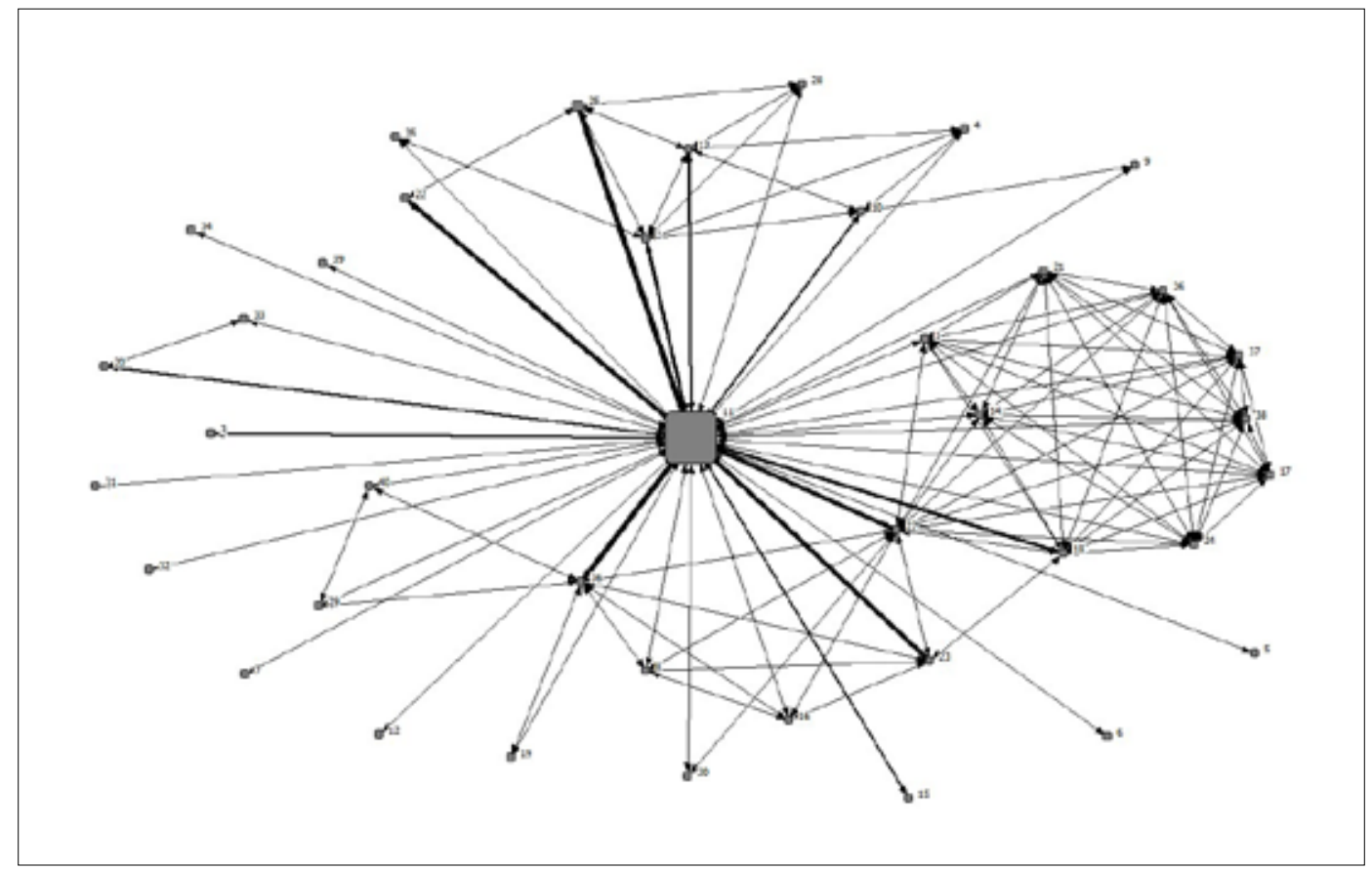

Fuente: elaboración propia 


\section{Conclusiones}

Los investigadores de las universidades catalanas publicaron el 60\% de los artículos sobre turismo en colaboración. La mayor parte de las colaboraciones fueron entre dos o tres autores. Durante la década, el porcentaje de colaboración aumentó, pero el número de autores por artículo no varió. En cuanto a las revistas, hubo diferencias en el porcentaje de colaboración y en el índice de coautoría. Y también las hubo entre las revistas indexadas y las no indexadas. Por otra parte, no se hallaron diferencias en la colaboración entre las revistas, ni entre los artículos, unidisciplinares y multidisciplinares. Hubo colaboración entre instituciones en el 31\% de los artículos. En el 8\% de los artículos hubo colaboración entre Cataluña y otras comunidades autónomas de España, y en el 12\%, entre Cataluña y otros países.

En relación al análisis de redes, en términos generales se puede hablar de una red dispersa o no conexa, con varias subredes. Destaca además el importante rol que juegan algunos autores e instituciones en la vinculación de la red. Sin embargo, no se pude negar la baja interrelación que en general existe entre los autores, máxime si se observa que el periodo estudiado comprende diez años, en los que varios de los autores han publicado un número muy reducido de artículos en coautoría. En este sentido, se debe resaltar la gran cantidad de autores aislados que, aunque no aparecen en el grafo (debido a que se eliminaron para facilitar el análisis), demuestran lo desconectada que se encuentra la red. Aunque también es importante considerar que por las características del tema analizado y tal como se menciona más adelante, difícilmente se podría tener una red completamente conectada.

Los resultados obtenidos son, hasta cierto punto, similares a los reportados por Benckendorff (2010), pues muestran un alto grado de dispersión y, simultáneamente, de agrupamiento. Situación que se hace evidente cuando se comparan los resultados con los reportados por otras disciplinas científicas. Otra coincidencia con el estudio referido es que los nodos (autores o instituciones) más productivos están generalmente más conectados y, además, son quienes frecuentemente lideran algunas de las subredes identificadas.

Así mismo, existe coincidencia con los resultados reportados por Ye et al. (2011), puesto que la red de colaboraciones de los autores catalanes parece ser muy holgada o flexible. Esto pudiera estar determinado, coincidiendo con los autores, por el hecho de que la investigación en turismo esté sufriendo un proceso de maduración y consolidación. O bien, debido a lo multidisciplinario del turismo, que resulta en una red de colaboración que incorpora muchos investigadores periféricos (de otras áreas o disciplinas), los cuales colaboran en proyectos esporádicos, con lo que los indicadores de la red disminuyen significativamente.

Por otro lado, el estudio abre líneas de investigación, como la posibilidad de ampliar el trabajo incorporando ciertos atributos, como podrían ser los grupos de investigación a los que pertenecen muchos de los autores analizados. Lo anterior, sin duda, daría luces para identificar si efectivamente estos grupos de investigación incentivan la producción científica o si, por el contrario, se constituyen más como unidades formales que funcionales.

\section{Bibliografía}

Albacete, Carlos A. y Fuentes, María del Mar

2010. "Difusión de la investigación española sobre turismo en revistas internacionales". Revista de Análisis Turístico, 9: 14-29.

Ardanuy, Jordi

2012. "Scientific collaboration in Library and Information Science viewed through the Web of Knowledge: the Spanish case". Scientometrics, 90(3): 877-890.

Barrios, Maite; Borrego, Ángel; Vilaginés, Andreu; Ollé, Candela y Somoza, Marta

2008. "A bibliometric study of psychological research on tourism". Scientometrics, 77(3): 453-467.

Beaver, Donald DeB.

2001. "Reflections on scientific collaborations (and its study): Past, present and prospective". Scientometrics, 52(3): 365-377.

Benckendorf, Pierre

2009. "Themes and trends in australian and new zealand tourism research: A social network analysis of citations in two leading journals (1994-2007)". Journal of Hospitality and Tourism Management, 16(1): 1-15. 
Benckendorf, Pierre

2010. "Exploring the limits of tourism research collaboration: A social network analysis of co-authorship patterns in Australian and New Zealand tourism research". Proceedings of the 20th Annual CAUTHE Conference, Hobart, Tasmania: 151-174.

Ceballos, Cristina; Arias, Carlos; Ruiz, Antonio; Sanz, Carlos y Vázquez, Isabel

2010. "La formación en turismo en España: pasado, presente y futuro en el nuevo Espacio Europeo de

Educación Superior". Cuadernos de Turismo, 25: 45-67.

Chang, Chia-Lin y McAleer, Michael

2012. "Citations and impact of ISI tourism and hospitality journals". Tourism Management Perspectives, 1: $2-8$.

De Solla Price, Derek J.

1966. Little Science, Big Science. Nueva York: Columbia University Press.

Direcció General de Turisme

2011. Catalunya turística en xifres 2010. Barcelona: Generalitat de Catalunya.

Eurostat

2011. Europe in figures. Eurostat yearbook 2011. Luxemburgo: Eurostat.

Faulkner, Herbert W.

2003. Progressing Tourism Research. Clevedon: Channel View.

Freeman, Linton C.

1978-1979. "Centrality in social networks: Conceptual clarification". Social Networks, 1(3): 215-239.

Garay, Luis A. y Cànoves, Gemma

2010. "Un análisis del desarrollo turístico en Cataluña a través del ciclo de evolución del destino turístico". Boletín de la Asociación de Geógrafos Españoles, 52: 43-58.

Glänzel, Wolfang y Schubert, András

2004. "Analysing scientific networks through coauthorship". En Moed, Henk F.; Glänzel, Wolfang y

Schmoch, Ulrich (Eds.). Handbook of quantitative science and technology research (pp. 257-276).

Netherlands: Springer.

González-Albo, Borja; Moreno, Luz; Aparicio, Javier; Morillo, Fernanda y Bordons, María

2009. La investigación española sobre turismo a través de publicaciones científicas de difusión nacional e internacional. IEDCYT, CCHS, CSIC. Documento no publicado.

Hernández, José M.; Campón, Ana M. y Folgado, José A.

2011. "La investigación en turismo en España a través de las revistas de dirección de empresas". Revista de Análisis Turístico, 12: 1-9.

Katz, J. Sylvan y Martin, Ben R.

1997. "What is research collaboration?". Research Policy, 26(1): 1-18.

McKercher, Bob; Law, Rob y Lam, Terry

2006. "Rating tourism and hospitality journals". Tourism Management, 27(6): 1235-1252.

Molina, José L.

2004. "La ciencia de las redes". Apuntes de Ciencia y Tecnología, 11: 36-42.

Pulido, Juan I. (Coord.)

2006. “Está la investigación en turismo suficientemente reconocida y valorada en España?” Revista de Análisis Turístico, 2: 103-112.

Racherla, Pradeep y Hu, Clark

2010. "A social network perspective of tourism research collaborations". Annals of Tourism Research, 37(4): 1012-1034.

Sánchez, Manuel y Marín, M. Belén

2003. "La investigación en turismo y economía de la empresa publicada en revistas especializadas españolas: 1996-2001". Papers de turisme, 33: 6-39.

Sonnenwald, Diane H.

2007. "Scientific collaboration". Annual Review of Information Science and Technology, 41(1): 643-681.

Torres, Anna

2010. "Sostenibilitat i modalitats turístiques. Una anàlisi de casos a Catalunya". Documents d'Anàlisi Geogràfica, 56(3): 479-502.

Xiao, Honggen y Smith, Stephen L.J.

2006. "The making of tourism research: Insights from a social sciences journal". Annals of Tourism Research, 33(2): 490-507. 
Ye, Qiang; Li, Tong y LAW, Rob

2013. "A coauthorship network analysis of tourism and hospitality research collaboration". Journal of Hospitality \& Tourism Research, 37(1): 51-76.

Zhao, Weibing y Ritchie, J.R. Brent

2007. "An investigation of academic leadership in tourism research: 1985-2004". Tourism Management, 28 (2): 476-490.

\section{Notas}

1 Thomson Reuters Institute for Scientific Information (http://ip-science.thomsonreuters.com/es/productos/wos).

2 Scopus de Elsevier (http://www.scopus.com).

3 Se publicaron 307 artículos en revistas españolas, pero no se dispuso de las disciplinas de 53 artículos. Por ello, este análisis se basa en 254 artículos.

4 Referido a los 307 artículos publicados en revistas españolas.

5 Referido a los 146 artículos publicados en revistas indexadas en Scopus.

6 La Tabla 4 desagrega la colaboración doméstica (es decir, entre autores de instituciones españolas) en dos niveles: colaboración catalana, cuando se produce entre autores de distintas instituciones sólo de Cataluña, y española, cuando se produce entre autores de instituciones catalanas y españolas.

7 Este porcentaje se refiere a la proporción que representan las colaboraciones (de Cataluña) con la comunidad autónoma en cuestión, respecto al total de colaboraciones con comunidades autónomas españolas.

8 Este porcentaje se refiere a la proporción que representan las colaboraciones (de Cataluña) con el país en cuestión, respecto al total de colaboraciones con países de fuera de España. 